\title{
Dermatopathia pigmentosa reticularis
}

INSERM

\section{Source}

INSERM. (1999). Orphanet: an online rare disease and orphan drug data base.

Dermatopathia pigmentosa reticularis. ORPHA:86920

A rare, genetic, ectodermal dysplasia characterized by a widespread, early-onset, reticulate hyperpigmentation that persists throughout life, mild, diffuse non-cicatricial alopecia, and onychodystrophy. There are no dental anomalies. Patients may also present with adermatoglyphia, palmoplantar hyperkeratosis, acral dorsal blistering, and hypohidrosis or hyperhidrosis. 